\title{
Departure Process of Ga from DLC Films Fabricated Using Ga Focused Ion Beam Assisted Deposition by Heat Treatment
}

\author{
Kazuhiro Kanda ${ }^{1}$, Makoto Okada ${ }^{1}$, Yuji Kang ${ }^{1}$, Tsuneo Suzuki ${ }^{2}$, and Shinji Matsui ${ }^{1}$ \\ ${ }^{1}$ University of Hyogo, Graduate School of Science, Laboratory of Advanced Science and Technology for Industry, \\ Kamigori, Hyogo 678-1205, Japan \\ Fax:81-791-58-0476, kanda@lasti.u-hyogo.ac.jp \\ ${ }^{2}$ Extreme Energy-Density Research Institute, Nagaoka University of Technology, Nagaoka, Niigata 940-2188, Japan \\ Fax:81-258-47-9890, suzuki@vos.nagaokaut.ac.jp
}

The departure process of residual Ga in diamond-like carbon (DLC) film synthesized by focused-ion-beam chemical vapor deposition (FIB-CVD) was investigated by measuring Rutherford backscattering (RBS) spectra of FIB-CVD DLC films after heat treatment in various conditions. The annealing temperature and annealing time were varied in the range of 473-973 $\mathrm{K}$ and $0-64 \mathrm{~h}$, respectively. The decrease in Ga concentration and transfer of Ga in the FIB-CVD DLC film were not observed due to heat treatment at less than $573 \mathrm{~K}$. The transfer of residual Ga from inside the film to the surface, and the formation of Ga spheres, which were surrounded by a graphite shell, were observed after heat treatment at $673 \mathrm{~K}$, but Ga concentration did not vary much. When the annealing temperature was higher than $773 \mathrm{~K}$, a decrease in the Ga concentration was observed. The depth profile of Ga in the DLC film in the RBS spectra was observed to divide into two peaks when treated with heat at temperatures higher than $673 \mathrm{~K}$. This splitting can be interpreted as the difference in the desorption rate of Ga in the DLC, graphite shell, and Si substrate. KEYWORDS: focused-ion-beam chemical-vapor deposition (FIB-CVD), diamond-like carbon (DLC), annealing effect, Rutherford backscattering spectrometry (RBS), thermal expansion

\section{INTRODUCTION}

A Ga focused-ion-beam chemical vapor deposition (FIB-CVD) method was developed as an effective technique for fabricating three-dimensional (3D) nanostructures [1]. Using this method, a pillar $80 \mathrm{~nm}$ in diameter can be fabricated by irradiating a focused $\mathrm{Ga}$ ion beam under a phenanthrene gas atmosphere, which was used as a carbon source, and a complex shape with an overhung, hollow, bridging structure can be formed by scanning a $\mathrm{Ga}$ ion beam. The fundamental structure of the carbon material formed by FIB-CVD is diamond-like carbon (DLC) by Raman spectroscopy [1] and near edge Xray absorption fine structure spectroscopy [2]. The Young's modulus of a pillar fabricated using the FIB-CVD technique was reported to exceed $600 \mathrm{GPa}[3,4]$, which is sufficiently large that nanodevices fabricated using this technique offer great possibilities in various applications, such as in the biochemical and electromechanical fields.

The pillar fabricated by FIB-CVD had a double structure such that the core region coexisted with $\mathrm{C}$ and residual Ga from the source ion and with its outer diamondlike carbon shell that did not contain $\mathrm{Ga}$. In addition, this residual $\mathrm{Ga}$ in the core- region transferred to the surface and produced $\mathrm{Ga}$ spheres there, which finally vaporized from the film by annealing at temperatures higher than 873 $\mathrm{K}[3,5,6]$. These movements of Ga were known to cause a change in the fundamental structure and several material properties, such as density [4], elemental composition [7], local structure [2, 8], Young modulus [3, 7] and hardness [8]. As noted above, the properties of nanodevices fabricated using the FIB-CVD technique can be controlled by heat treatment. However, the details on the departure process of Ga in the FIB-CVD DLC film have not been fully understood. We therefore investigated the behavior of $\mathrm{Ga}$ atom in FIB-CVD DLC films during annealing. Sample films fabricated using the FIB-CVD method were annealed under various conditions, such as annealing temperatures in the range of 473-973 $\mathrm{K}$ and annealing times in the range of 0-64 h. The Ga concentration and depth profile of FIBCVD DLC films after heat treatment were measured using Rutherford backscattering spectrometry (RBS) techniques.

\section{Experimental}

An FIB-CVD DLC film was formed on a $\mathrm{Si}$ substrate surface using a commercially available FIB-CVD system (SMI2050MS: SII Nano Technology Inc.). The details of the method used to fabricate the FIB-CVD DLC films are described in a previous paper [1,9]. Briefly, the accelerating voltage of a $\mathrm{Ga}^{+}$ion beam and ion beam currents were at $30 \mathrm{keV}$ and $28 \mathrm{nA}$, respectively. The system was equipped with two gas nozzles; a source gas, phenanthrene $\left(\mathrm{C}_{14} \mathrm{H}_{10}\right)$, was supplied through these nozzles, and phenanthrene molecules were adsorbed on the $\mathrm{Si}$ substrate. The base pressure of the sample chamber was $9 \times$ $10^{-5} \mathrm{~Pa}$, and the chamber pressure during introduction of the source gas was $1 \times 10^{-4} \mathrm{~Pa}$. The FIB was scanned to write a square pattern $200 \mathrm{~m} \times 200 \mathrm{~m}$ using a computercontrolled scanning system. The produced FIB-CVD DLC was covered with these squares, resulting in a total area that $5 \times 5 \mathrm{~mm}^{2}$. We intended to deposit FIB-CVD films with thickness of a $200 \mathrm{~nm}$. The actual thickness of the formed individual films was estimated using a surface profiler (ULVAC: DEKTAK 6M). The formed FIB-CVD DLC films were annealed in a vacuum using a furnace (ThermoRIKO: GFA430). The back pressure and pressure during annealing in the furnace were $\approx 1 \times 10^{-5}$ and $\approx 5 \times 10^{-5}$ 
$\mathrm{Pa}$, respectively. The annealing temperature ranged from $473 \mathrm{~K}$ to $973 \mathrm{~K}$, and the annealing time ranged from 2 to 64 h. We observed the surface of the FIB-CVD DLC film and roughly estimated the $\mathrm{Ga} / \mathrm{C}$ ratio using a scanning electron microscope energy dispersive $\mathrm{x}$-ray spectrometer (SEMEDX) (JEOL: JSM6700F).

An RBS measurement with $\mathrm{MeV}-\mathrm{He}^{+}$irradiation was performed using an electrostatic accelerator (NisshinHigh Voltage: NT-1700HS) at the Extreme Energy Density Research Institute, Nagaoka University of Technology. The details of RBS measurement are described in refs. 9-11. Briefly, the $\mathrm{He}^{+}$ions accelerated to $2.5 \mathrm{MeV}$ were used as incident beam at $72^{\circ}$ with respect to the surface normal of samples. $\mathrm{A} \mathrm{He}^{+}$beam current was maintained at $6 \mathrm{nA}$. A small fraction, $\sim 0.1 \%$, of high-energy $\mathrm{He}^{+}$ions scattered elastically by the sample were captured with a solid-state detector (SSD) arranged at $12^{\circ}$ with respect to the surface normal of samples toward the incident beam. No signal, except for signals corresponding to $\mathrm{C}, \mathrm{Ga}$, and $\mathrm{Si}$, which was due to the substrate, was observed in the RBS spectra. The content of these atoms was determined using the RBS fitting calculation package (Nissin High Voltage Co.: ERNIE ver. 1.0) on the basis of film thickness, estimated with a profiler, and $\mathrm{Ga} / \mathrm{C}$ ratio, estimated with the SEMEDX. The estimation error was 0.5 at. $\%$, because the content examination was simulated at 1 at.\% steps.

\section{Results and discussions}

The dependence of the $\mathrm{Ga} / \mathrm{C}$ ratio on annealingtime is plotted in Fig. 1. The $\mathrm{Ga} / \mathrm{C}$ ratio in the as-deposited FIB-CVD DLC film was $\approx 4$ at. $\%$. The Ga concentration in the film is expected to be slightly different in each film, because the deposition conditions were not identical. The Ga concentration in the FIB-CVD DLC film maintained its initial value after the annealing at $573 \mathrm{~K}$. The annealing at $673 \mathrm{~K}$ caused a slight decrease in the Ga concentration in the annealing time range from 0 to $16 \mathrm{~h}$ and a slight increase from 16 to $32 \mathrm{~h}$. These variations can be considered as a small desorption of Ga from the DLC surface, and the formation of Ga spheres on the DLC surface, as described below. On the other hand, the Ga concentration decreased remarkably by annealing at temperatures higher than $773 \mathrm{~K}$ with an increase in the annealing time. The desorption temperature of $\mathrm{Ga}$ from the

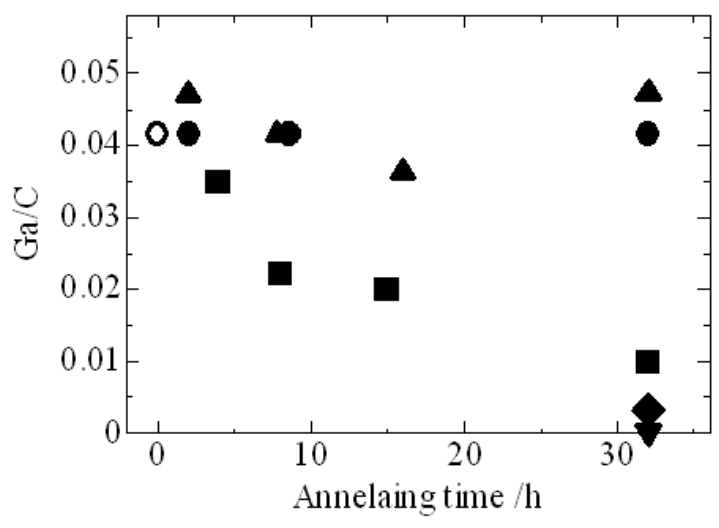

Fig. 1 Dependence of $\mathrm{Ga} / \mathrm{C}$ ratio of FIB-CVD DLC film on annealing time determined by the RBS measurements. Open circle indicates $\mathrm{Ga} / \mathrm{C}$ ratio of FIBCVD DLC film as deposition. Closed circles, closed triangles, and closed squares indicate the $\mathrm{Ga} / \mathrm{C}$ ratios of FIB-CVD DLC films after heat treatment at 573, 673, and $773 \mathrm{~K}$, respectively. Closed inverse triangle and closed diamond indicate the $\mathrm{Ga} / \mathrm{C}$ ratios of FIB-CVD DLC films after $32 \mathrm{~h}$ of heat treatment at 873 and 973 K. resnectivelv.
FIB-CVD DLC film agreed well with the thermal desorption spectroscopy (TDS) study [6].
$\mathrm{Oh}$
$4 \mathrm{~h}$
$15 \mathrm{~h}$
$64 \mathrm{~h}$

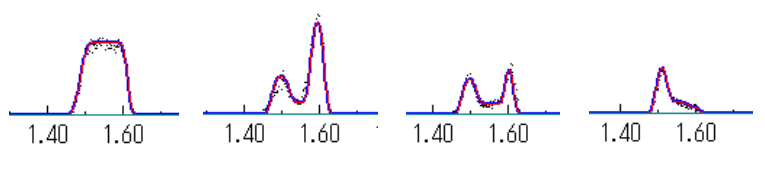

Energy of back-scattered $\mathrm{He}^{+} / \mathrm{MeV}$

Fig. 2 Ga profiles in the RBS spectra of FIB-CVD DLC films as- deposited and after heat treatment at 773 $\mathrm{K}$. Annealing times are noted in the upper part of each spectrum. The plots indicate the measured RBS spectra, and the solid lines indicate the fitting curves reproduced using the RBS fitting calculation package.

Figure 2 depicts the Ga profiles in the RBS spectra of the FIB-CVD DLC film (a) as deposited, and annealed FIB-CVD DLC film at $773 \mathrm{~K}$ for (b) $2 \mathrm{~h}$, (c) $15 \mathrm{~h}$, and (d) $64 \mathrm{~h}$. The $\mathrm{X}$ - axis indicates the energy of $\mathrm{He}^{+}$ion backscattered by the Ga in the FIB-CVD DLC film; namely, the high energy side and low energy side correspond to the Ga atoms near the surface and $\mathrm{Ga}$ atoms at the bottom of the film, respectively. The Ga profile of the FIB-CVD DLC film as- deposited (a) has a rectangular shape, which indicates that $\mathrm{Ga}$ atoms were distributed uniformly. However, the Ga profile in the FIB-CVD DLC film was divided into peaks by the $2-\mathrm{h}$ heat treatment at $773 \mathrm{~K}$ (b). In other words, the concentration of $\mathrm{Ga}$ in the middle part of the film decreased earlier than at the near-surface part and bottom part of the film. The peak intensity of the nearsurface part was larger than that of the bottom part in the film after $2 \mathrm{~h}$ of heat treatment at $773 \mathrm{~K}$ (b), while the peak intensity of the near-surface part was less than that of the bottom part in the film after $15 \mathrm{~h}$ of heat treatment at $773 \mathrm{~K}$ (c). After $64 \mathrm{~h}$ of heat treatment at $773 \mathrm{~K}$, only a small amount of Ga was left, in the bottom part (d). These Ga profiles indicates that the desorption rate of $\mathrm{Ga}$ increased in the order of the middle part, upper part, and bottom part.

Some studies have reported that $\mathrm{Ga}$ segregated from an FIB-CVD device by annealing treatment formed Ga spheres by agglomerating [3, 5, 12]. These Ga spheres were surrounded by a graphite shell. Figure 3 shows an SEM image of the DLC film after $32 \mathrm{~h}$ of heat treatment at $673 \mathrm{~K}$. The squares with $200-\mathrm{m}$ sides indicate the deposition area of one scan of the FIB beam, as described in the experimental section. A lot of $\mathrm{Ga}$ spheres were observed on the surface of the FIB-CVD DLC film. The increase in $\mathrm{Ga}$ concentration after $32 \mathrm{~h}$ of heat treatment at $673 \mathrm{~K}$ in Fig. 1 can be attributed to the formation of these $\mathrm{Ga}$ spheres; in other words, a lot of Ga existed near the surface, where the detectivity of the RBS apparatus is higher. The slight decrease in Ga concentration of the FIBCVD film after heat treatment in the range of $0-16 \mathrm{~h}$ at 673 $\mathrm{K}$ indicated that the $\mathrm{Ga}$ atoms from the inner part of the FIB-CVD film transferred to the surface, and a small quantitty of them departed. The desorption rate from a $\mathrm{Ga}$ sphere is thought to be smaller than that from DLC, namely amorphous carbon, because the $\mathrm{Ga}$ spheres were surrounded by a graphite shell, which was composed of a large mount of graphite, namely crystalline carbon. On the other hand, the bottom part, where some Ga remained after long heat treatment, is thought to be the Ga-implanted $\mathrm{Si}$ substrate. The desorption rate of $\mathrm{Ga}$ from this region was expected to be smaller than that from the DLC, because the 
diffusion constant of the Si substrate is smaller than that of DLC, even if the Si substrate is damaged by the $\mathrm{Ga}$ ion beam.

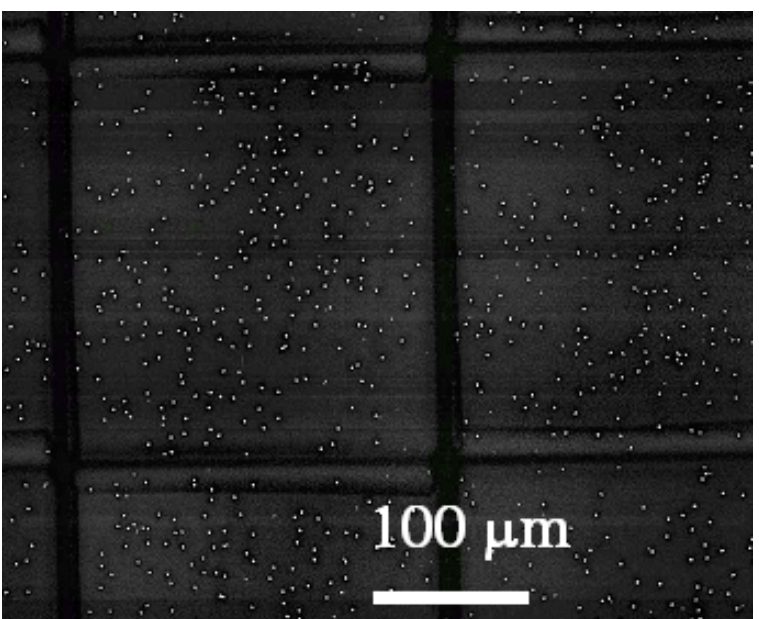

Fig. 3 SEM image of FIB-CVD DLC film after $32 \mathrm{~h}$ of heat treatment at $673 \mathrm{~K}$.

We can summarize the departure process of Ga from the FIB-CVD DLC film as follows. Figure 4 shows a schematic illustration of the annealing effect on the FIBCVD DLC film. In the chemical vapor deposition by FIB, the $\mathrm{Ga}$ was implanted into the Si substrate. The ion range of 30- $\mathrm{kV}$ Ga ions into the $\mathrm{Si}$ substrate was calculated to be $\approx 35 \mathrm{~nm}$ by Monte Carlo simulation [13]. On the other hand, the DLC layer, which contains no $\mathrm{Ga}$, is formed near the surface. The thickness of this region is $\approx 35 \mathrm{~nm}[4,6]$. Therefore, the structure of the FIB-CVD DLC film deposited on the Si substrate was consisted of four kinds of layers, a DLC layer $\approx 35 \mathrm{~nm}$ in thickness neighbouring the surface without Ga, a DLC layer containing Ga in the middle part, Ga-implanted $\mathrm{Si}$ substrate of $\approx 35 \mathrm{~nm}$ thick, and a $\mathrm{Si}$ substrate without damage (Fig. 4(a)). The heat treatment at $\approx 673 \mathrm{~K}$ caused residual $\mathrm{Ga}$ to expand. Ga atoms in the inner part of the film then transferred to the surface (Fig. 4(b)). The Ga segregated by the annealing
(A)

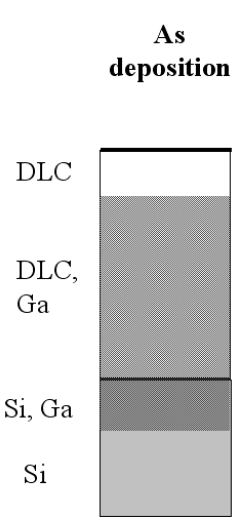

(B)

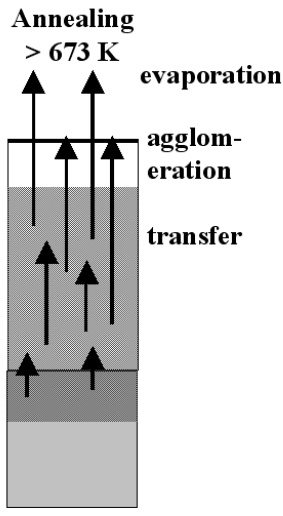

(C)

Ga sphere

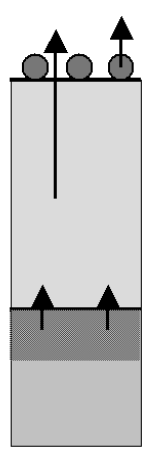

Fig. 4 Schematic illustration of Ga departure from FIB-CVD DLC film. (A) FIB-CVD DLC film as- deposition. (B) With heat treatment at temperatures higher than $673 \mathrm{~K}$, residual $\mathrm{Ga}$ transferred toward the surface and agglomerated into Ga spheres. (C) Ga concentration in the middle part of the FIB-CVD DLC film decreased, because the desorption rate decreased in the order of DLC, graphite shell, and Si substrate. treatment formed $\mathrm{Ga}$ spheres by agglomerating. The desorption rate increased sequentially starting with the $\mathrm{Si}$ substrate, inner graphite shell, and then the inner DLC (Fig. 4(c)). Therefore, the Ga concentration first decreased in the middle part of film, which was composed of DLC, then in the upper part, where Ga spheres surrounded by a graphite shell were formed. Finally, the Ga departed from the bottom part, where $\mathrm{Ga}$ ions were implanted in the $\mathrm{Si}$ substrate.

In conclusion, we investigated the departure process of $\mathrm{Ga}$ after applying an FIB-CVD process by preparing samples at various annealing temperatures and annealing times. The Ga atoms in the FIB-CVD DLC film did not move at heat treatment of $523 \mathrm{~K}$. At $673 \mathrm{~K}$, residual $\mathrm{Ga}$ transferred to the surface and formed Ga spheres, although the evaporation rate was very low. With heat treatment at temperatures higher than $773 \mathrm{~K}$, the residual $\mathrm{Ga}$ was evaporated, and the Ga concentration decreased as the annealing time increased.

\section{Acknowledgments}

A part of this work was supported by the New Energy and Industrial Technology Development Organization (NEDO) under the work entitled "Advanced Fundamental Research Project on Hydrogen Storage Materials" and Grant-in-Aid for Science Research from the Ministry of Education, Culture, Sports, Science and Technology of Japan under Contact No. 20310068. We thank Mr. Katagiri, Mr. Sekimoto, and Mr. Obata of the Extreme Energy Density Research Institute, Nagaoka University of Technology for the RBS measurements.

\section{References}

[1] S. Matsui, T. Kaito, J. Fujita, M. Komuro, K. Kanda, and Y. Haruyama, J. Vac. Sci. Technol. B, 18, 3181-3184 (2000).

[2] K. Kanda, J. Igaki, Y. Kato, R. Kometani, and S. Matsui, Radiat. Phys. Chem. 75, 1850-1854 (2006).

[3] J. Fujita, M. Ishida, T. Ichihashi, T. Sakamoto, Y. Ochiai, T. Kaito, and S. Matsui, Jpn. J. Appl. Phys., 41, 4423-4426 (2002).

[4] J. Fujita, M. Ishida, T. Ichihashi, Y. Ochiai, T. Kaito, and S. Matsui: Nucl. Instrum. Methods Phys. Res. B, 206, 472-477 (2003).

[5] R. Kometani, S. Ishihara, K. Kanda, Y. Haruyama, T. Kaito, and S. Matsui, Jpn. J. Appl. Phys., 47, 5032-5035 (2008).

[6] T. Nagata, Y. Sakuma, M. Haemori, K. Nakajima, R. Kometani, K. Kanda, S. Matsui, and T. Chikyow, Jpn. J. Appl. Phys., 47, 9010-9012 (2008).

[7] K. Kanda, J. Igaki, A. Saikubo, R. Kometani, T. Suzuki, K. Niihara, H. Saitoh, and S. Matsui, Jpn. J. Appl. Phys., 47, 7467-7469 (2008).

[8] A. Saikubo, K. Kanda, Y. Kato, J. Igaki, R. Kometani, and S. Matsui, Jpn. J. Appl. Phys., 46, $7512-7513$ (2007).

[9] J. Igaki, A. Saikubo, R. Kometani, K. Kanda, T. Suzuki, K. Niihara, and S. Matsui, Jpn. J. Appl. Phys., 46, 80038004 (2007)

[10] Y. Ohkawara, S. Ohshio, T. Suzuki, H. Ito, K. Yatsui, and H. Saitoh, Jpn. J. Appl. Phys., 40, 3359-3363 (2001).

[11] Y. Ohkawara, S. Ohshio, T. Suzuki, H. Ito, K. Yatsui, and H. Saitoh, Jpn. J. Phys., 40, 7007-7012 (2001).

[12] R. Kometani, T. Ichihashi, S. Warisawa, and S. Ishihara, Jpn. J. Appl. Phys., 48, 06FE01-4 (2009).

[13] R. Kometani, Ph. D. Thesis, University of Hyogo, (2007) 31-37.

(Received September 14, 2009; Accepted February 16, 2011) 